\title{
Passive and active light scattering obstacles
}

\section{Maris Ozolinsh, Juan Bueno, Varis Karitans, Gatis Ikaunieks}

Maris Ozolinsh, Juan M. Bueno, Varis Karitans, Gatis Ikaunieks, "Passive and active light scattering obstacles," Proc. SPIE 9664, Ninth International Topical Meeting on Education and Training in Optics and Photonics, 96640J (24 October 2005); doi: 10.1117/12.2207740

SDIE Event: Ninth International Topical Meeting on Education and Training in Optics and Photonics, 2005, Marseille, France 
Ref ETOP072

\title{
Passive and active light scattering obstacles
}

\author{
Maris Ozolinsh ${ }^{a}$, Juan M. Bueno ${ }^{b}, V^{2}$ aris Karitans ${ }^{c}$, and Gatis Ikaunieks ${ }^{c}$
}

${ }^{a}$ Institute of Solid State Physics, University of Latvia

8 Kengaraga Str., LV-1063 Riga, LATVIA

'Laboratorio de Óptica (Dpto. Física), Universidad de Murcia

Campus de Espinardo (Edificio C), 30071, Murcia, SPAIN

${ }^{\mathrm{C}}$ Department of Optometry and Vision Science, University of Latvia

8 Kengaraga Str., LV-1063 Riga, LATVIA

ozoma@latnet.Iv

\begin{abstract}
s
Simulation of vision pathologies and adverse viewing conditions in laboratory conditions requires optical phantoms with different level of light scattering. Such obstacles are designed as passive or active elements applying several technologies. We used for studies two kinds of solid state smart materials with electrically controllable light scattering - electrooptic PLZT ceramics, polymer dispersed liquid crystals PDLC and obstacles with fixed light scattering composite of polymer methylmethaacrilat PMM together with grinded glass microparticles. Report analyzes optical characteristics of such obstacles - attenuation, scattering, depolarization of different wavelength light at various scattering levels and changes of visual performance applying obstacles in vision science studies.
\end{abstract}

\section{Key words}

light scattering, light polarization, polymer dispersed liquid crystals, birefringence, visual perception

\section{Summary}

Simulation of vision pathologies and adverse viewing conditions in laboratory conditions requires optical phantoms with different level of light scattering. Such obstacles can be designed as passive or active elements applying several technologies. We have used for such purposes two kinds of solid state smart materials with electrically controllable light scattering - electrooptic PLZT ceramics, polymer dispersed liquid crystals PDLC ${ }^{2}$ and obstacles with fixed light scattering composite of polymer methylmethaacrilat PMM together with grinded glass microparticles.

PLZT ceramics (composition similar to piezoelectric PZT - lead zirconate titanate, however in our case additionally modified by

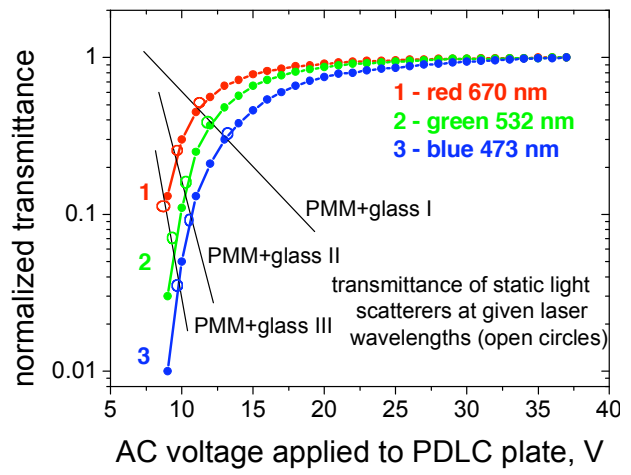

Fig.2. Transmittance of PLDC cell with AC voltage applied at three RGB laser wavelengths $670 \mathrm{~nm}$ (upper curve), $532 \mathrm{~nm}$ (middle) and $473 \mathrm{~nm}$ (lower curve). 
lanthanum), is a transparent in visible bulk ferroelectic polycrystalline material. Different scattering levels were generated by changing the voltage applied to the ceramics. In absence of electric field applied in our devices ceramic compositions stay uniform and nonpolar. When an electrical field is applied to transparent electrodes deposited on the opposite sites of the $1.5 \mathrm{~mm}$ thick PLZT plate, a reversible nucleation of submicron size dielectrically polar and thus also birefringent regions are induced, thus producing local variations of the refractive index in ceramics. That produces an amount of forward straylight that increases with voltage.

PLDC cells used in experiments (Fig.1) as eye obstacles consist of two glass plates with transparent ITO electrodes forming a 10 microns gap of a composite polymer (PN393 MerckKgaA) with dispersed liquid crystal (BL035 MerckKgaA) droplets of micrometers size. Values of the refractive index were - for polymer $n=1.473$ (at $589 \mathrm{~nm}$ ) and for birefringent liquid crystal $n_{o}=1.528$ and $n_{e}$ same as for polymer. Applying the $A C$ voltage aligns directors of liquid crystal droplets along the direction of the electric field $\boldsymbol{E}$ in the layer - light passing the cell does not meet refractive index variations, and no scattering takes place. At absence
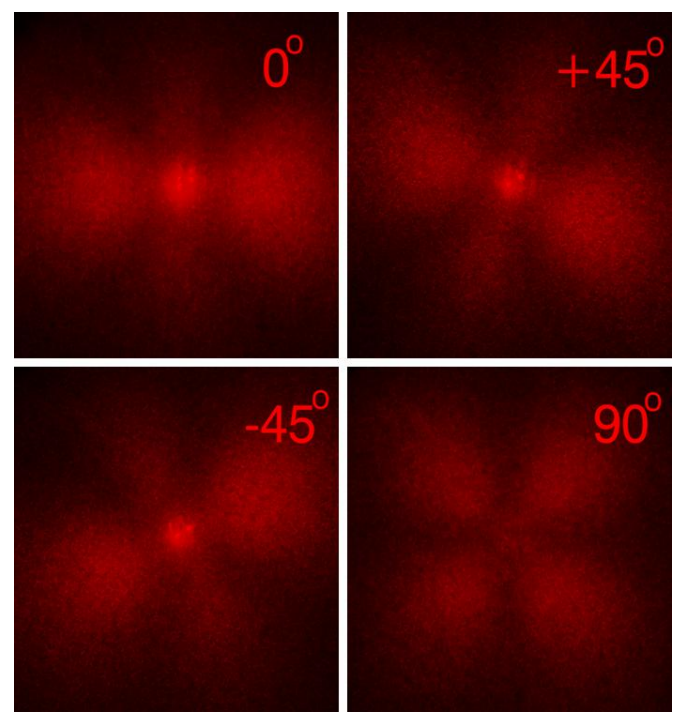

Fig.4. Indicatrices of the scattered light by PDLC light scattering obstacles analyzing it with a rotating polarizer. Orientation of analyzer corresponding to the polarization plane of the initial $633 \mathrm{~nm}$ laser beam is shown at the right lig upper part of images.
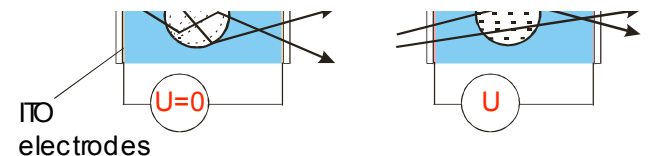

appearance milky of an external influence droplets are randomly oriented causing local optical non-homogeneities and light scattering. As the birefringence is much higher as compared with PLZT ceramics the thickness of the scattering layer for similar scattering degrees is within a micrometer range. Thus the voltage $U$ needed maximally to align liquid crystal droplet directors along the transmitted light beam does not override 30-40 V. In such PDLC plate Mie light scattering takes place opposite to PLZT ceramics that due to smaller size of light scattering birefringent domains can be characterized with Rayleigh scattering. However also for Mie scattering in PDLC the noticeable wavelength dependence is observed. Figure 2 shows the attenuation of the collimated directly transmitted light beams at three wavelengths (red, green and blue) of the RGB laser.

Fig.1. Polymer disperse liquid crystal cell in two states - light scattering Off state and transparent On state. 


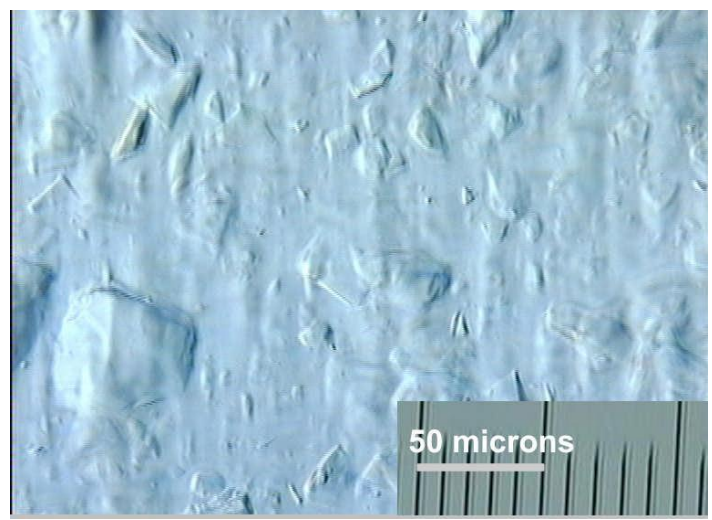

Fig.3. Interference contrast microscopy of glass particles in PMMA matrix.
Another way to obtain graded stepby-step light scattering is using of composite - matrix of less refractive glue monomer of methaacrilate MMA (refractive index $\mathrm{n}=1.45$ ) mixed together with dispersed small glass particles $(n=1.5)$. A gap between two glass plates of thickness $0.06-0.5$ microns are filled with such mixture (glass powder concentration 100-200 $\mathrm{mg} / \mathrm{ml}$ ) thus obtaining pairs of obstacles with fixed but graded degrees of the light scattering. A picture of an interference contrast microscopy is depicted in Fig.3. Also for such scattering obstacles scattered light is strongly wavelength dependent in visible spectral range (Fig.2).

Besides intensity and spectral dependencies the scattered light produced by such obstacles reveals also noticeable polarization dependencies. Figure 4 shows polarimetry analysis of the scattered by PDLC cell linear polarized $633 \mathrm{~nm}$ laser light applying the rotating analyzer. Obtained pictures allow to analyze polarimetry of the scattered light for angles much larger as those determining normal visual acuity. However previously Bueno at all. ${ }^{3,4}$ have analyzed light scattering in human eyes and have revealed similarity of changes of the depolarization degree produced by such scatterers (PLZT ceramics) comparing it with the depolarization degree of the scattered light in elder cataract eye patients at smaller angles - below 1 arc degree.

All kind of such light scattering obstacles have been used for determination of various visual performance characteristics. Visual acuity looking through such obstacles with graded scattering drops down from standard 20/20 to zero for black-white and black-red, black-green and black-blue stimuli (for black-blue the most drastic between all listed optotypes). Scattering obstacles are used in vision research also for studies of colour contrast sensitivity, stimuli recognition search time and diminishing of objective optical characteristics of the model eye in presence of scattering. Studies of visual performance of observers without any vision pathologies however using obstacles capable to induce different degrees of light scattering allow to simulate a decrease of visual functions for cataract persons ${ }^{5}$ and also decrease of recognition of colour stimuli in adverse weather conditions.

\section{References.}

1. Ozolinsh M., Lacis I., Paeglis R., Sternberg A., Svanberg S., Andersson-Engels S., and Swartling, J. (2002), Electrooptic PLZT ceramics devices for vision science applications, Ferroelectrics, 273, pp.131-136.

2. Ozolinsh M., Korenuks D., Kozachenko A., and Komitov L. (2003), Light scattering effect on colour stimuli visual response, Perception, 32, S178-179.

3. Bueno J.M. (2001), Depolarization effects in the human eye Vision Res. 41 2687-96.

4. Bueno J.M., Berrio E., Ozolinsh M., and Artal P. (2004), Degree of polarization as an objective method of estimating scattering, J. Opt. Soc. Am. A, 21, pp.1316-1321.

5. Ozolinsh M. and Papelba G. (2004), Eye cataract simulation using polymer dispersed liquid crystal scattering obstacles, Ferroelectrics, 304, pp.207-212. 